\title{
Subthalamic Nucleus Stimulation in Pediatric Isolated Dystonia: A 10-Year Follow-up
}

\author{
Wenying Xu*, Hongxia Li*, Chencheng Zhang, Bomin Sun, Yiwen Wu, Dianyou Li
}

\begin{abstract}
Objective: To evaluate the short-term and long-term clinical effectiveness and safety of subthalamic nucleus deep brain stimulation (STN-DBS) for medically intractable pediatric isolated dystonia. Methods: Using a longitudinal retrospective design, we assessed the clinical outcomes of nine patients who underwent STN-DBS for treatment-refractory pediatric isolated dystonia one decade ago (mean age at surgery: $15.9 \pm 4.5$ years). The primary clinical outcome used was assessed by retrospective video analyses of patients' dystonia symptoms using the Burke-Fahn-Marsden Dystonia Rating Scale (BFMDRS). Clinical assessments were performed at baseline, 1-year follow-up (1-yr FU), and 10-year follow-up (10-yr FU). Adverse side effects, including surgery-related, device-related, and stimulation-related effects, were also documented. Results: After STN-DBS surgery, the mean improvement in the BFMDRS motor score was $77.1 \pm 26.6 \%$ at 1 -yr FU and $90.4 \pm 10.4 \%$ at $10-y r$ FU. Similarly, the mean BFMDRS disability score was improved by $69.5 \pm 13.6 \%$ at $1-y r$ FU and by $86.5 \pm 13.9 \%$ at $10-y r$ FU. The clinical improvements gained at 10 -yr FU were significantly larger than those observed at 1-yr FU. Negative correlations were found between the duration of disease to age at surgery ratio (DD/AS) and the improvements in the BFMDRS motor score and total score at 1-yr FU and 10-yr FU. Conclusion: To our knowledge, this study provides the first clinical evidence for the short- and long-term effectiveness and safety of STN-DBS for pediatric isolated dystonia. Additionally, putative evidence is provided that earlier STN-DBS intervention in patients with refractory pediatric isolated dystonia may improve short- and long-term clinical outcomes.
\end{abstract}

RÉSUMÉ : Stimulation du noyau sous-thalamique d'enfants atteints de dystonie isolée : un suivi sur dix ans. Objectif : Évaluer l'efficacité clinique à court et à long terme de la stimulation cérébrale profonde (SCP) du noyau sous-thalamique, ainsi que sa sécurité, dans le cas d'enfants atteints de dystonie isolée réfractaire. Méthodes : Au moyen d'un modèle longitudinal rétrospectif, nous avons cherché à évaluer les résultats cliniques de 9 jeunes patients atteints de dystonie isolée ayant été soumis à une SCP du noyau sous-thalamique il y a une décennie (âge moyen au moment de l'intervention chirurgicale : 15,9 \pm 4,5 ans). Le principal résultat clinique que nous avons utilisé a été évalué au moyen de la Burke-Fahn-Marsden Dystonia Rating Scale (BFMDRS) et d'analyses vidéo rétrospectives des symptômes de ces patients. Nos évaluations cliniques ont été ensuite menées tout de suite après une intervention de SCP, lors d'un suivi 12 mois plus tard et finalement lors d'un suivi effectué au bout de 10 ans. Nous avons également documenté les effets indésirables liés à la SCP, aux appareils et aux stimulations proprement dit. Résultats : À la suite d'une SCP du noyau sous-thalamique, l'amélioration moyenne des scores moteurs à la BFMDRS a été de 77,1 $\pm 26,6 \% 12$ mois plus tard et de 90,4 $\pm 10,4 \%$ au bout de 10 ans. De la même manière, le score moyen d'invalidité à la BFMDRS a été amélioré de 69,5 $\pm 13,6 \% 12$ mois plus tard et de 86,5 $\pm 13,9 \%$ au bout de 10 ans. Les améliorations cliniques notées après 10 ans se sont quant à elles révélées nettement plus importantes que celles observées après seulement 12 mois. À noter que des corrélations négatives ont été constatées entre la durée de la maladie et le ratio de l'âge à l'intervention (age at surgery ratio) ainsi qu'entre les améliorations aux scores moteurs à la BFMDRS et les scores totaux après 12 mois et 10 ans. Conclusion : À notre connaissance, cette étude fournit les premières preuves cliniques de la sécurité et de l'efficacité à court et à long terme de la SCP du noyau sous-thalamique dans le cas d'enfants atteints de dystonie isolée. De plus, nous avons soumis des preuves qu'une SCP du noyau sous-thalamique plus précoce pourraient améliorer les résultats cliniques de ces patients à court et à long terme.

Keywords: Isolated dystonia, Pediatric dystonia, Subthalamic nucleus, Deep brain stimulation, Long-term effects doi:10.1017/cjn.2020.32

Can J Neurol Sci. 2020; 47: 328-335

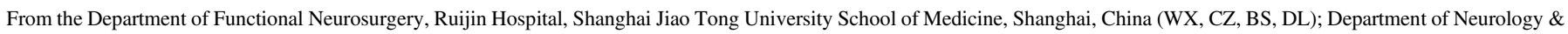
Institute of Neurology, Ruijin Hospital, Shanghai Jiao Tong University School of Medicine, Shanghai, China (HL, YW)

Received July 31, 2019. Final Revisions Submitted February 5, 2020. Date of Acceptance February 8, 2020

*Theses authors contributed equally to this work.

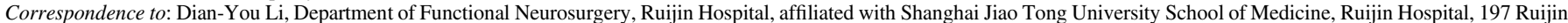

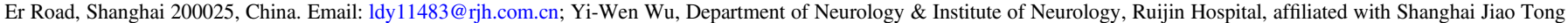
University School of Medicine, Shanghai, China. Email: wyw11380@rjh.com.cn 


\section{INTRODUCTION}

Dystonia is a movement disorder characterized by uncoordinated or excessive contractions of the agonist and antagonist muscles, leading to persistent or intermittent involuntary and repetitive aberrant movements, body postures, or both. Based on associated features (additional movement disorders or neurological features), dystonia may be divided into isolated dystonia and combined dystonia. ${ }^{1}$ In isolated dystonia, dystonia is the only motor symptom, with the exception of tremor. In combined dystonia, by contrast, dystonia is combined with other movements disorders, such as myoclonus.

Dystonia with an onset in childhood (3-12 years) or adolescence (13-20 years) often becomes generalized over the illness course, with a progressive increase in the number of affected body parts. A recent study of pediatric patients reported that dystonia symptoms often worsen during development, with one in three cases remaining symptomatic stable at most. ${ }^{2}$ Moreover, childhood dystonia when left untreated or when treated unsuccessfully may lead to irreversible fixed bone deformation and disability. ${ }^{3}$ At present, however, limited options are available for the clinical management of pediatric dystonia. A main option is pharmacotherapy, but this treatment may induce adverse side effects and affect growth and development. ${ }^{4}$ Furthermore, some patients do not clinically respond to medication or other standard treatments. One therapeutic intervention that seems promising for managing medically intractable pediatric dystonia is deep brain stimulation (DBS).

In adult patients, DBS targeting the globus pallidus interna (GPi) has already been successfully used in the management of dystonia. ${ }^{5-7}$ The GPi is part of a cortico-striato-pallido-thalamo-cortical loop that has traditionally been implicated in the pathogenesis and pathophysiology of dystonia. ${ }^{8}$ Several studies have also indicated that GPi-DBS can reduce the severity of dystonia in pediatric patients. ${ }^{9-11}$ In one of these studies, however, it was observed that although GPi-DBS greatly improved dystonia symptoms in two pediatric patients initially, the symptoms of each patient gradually reappeared within a year after surgery. ${ }^{10}$ This observation raises the possibility that the GPi may not invariably be the clinically optimal target in DBS treatment for pediatric dystonia. Indeed, the basic lessons learned from DBS treatment studies of other movement disorders, such as Parkinson's disease, are that the identification of the appropriate target is crucial, that different targets within a certain network can have different clinical benefits and adverse-event profiles, and that the specific target used does not necessarily have to be the same across and within patients. ${ }^{12,13}$ In the present study, therefore, we explored the utility of a target other than the GPi in managing treatment-refractory pediatric dystonia.

In this retrospective study, we examined the clinical outcomes of several patients who had undergone STN-DBS surgery for medically intractable pediatric isolated dystonia about one decade ago. Like the GPi, the STN is also a key structure within the cortico-striato-pallido-thalamo-cortical loop implicated in the pathophysiology of dystonia. Although the clinical utility of STN-DBS for pediatric dystonia has not yet been explored, the STN is considered an optimal stimulation target for isolated dystonia in adults. ${ }^{14}$ Also, our medical center and multidisciplinary team have a long history of treating dystonia with STN-DBS, ${ }^{15,16}$ including repeated follow-up assessment, clinical care, and longterm maintenance. ${ }^{14}$ Here, we provide for the first time clinical evidence supporting the short- and long-term effectiveness and safety of STN-DBS for pediatric isolated dystonia.

\section{Methods \\ Participants}

Participating patients were recruited by retrospectively reviewing the clinical histories, diagnoses, treatments, clinical outcomes, and adverse side effects from all patients documented in our hospital's computerized patient record system. To be eligible, they had to meet the following surgical inclusion criteria: (1) clinical diagnosis of dystonia either with or without a positive family history based on clinical diagnostic assessments and a family history method; (2) dystonia refractory to oral medications and to chemodenervation with botulinum toxin; (3) significant functional impairment; (4) age at illness onset younger than 18 years; and (5) younger than 21 years at the time of STN-DBS surgery. ${ }^{17}$ Surgical exclusion criteria were (1) dystonia due to an acquired cause; (2) contradiction to neurosurgery or anesthesia; and (3) a follow-up duration less than 12 months. All patients underwent genetic testing of the DYT-1 and DYT-6 loci pre- or post-operatively. The clinical protocol was approved by the ethics committee of the Ruijin Hospital Shanghai Jiao Tong University School of Medicine. Each patient and his or her family member provided written informed consent for study participation, including reporting the results without personally identifiable information.

\section{Surgical Procedure}

All pediatric patients had received bilateral STN-DBS surgery performed by the same neurosurgeons from the Department of Functional Neurosurgery in Ruijin Hospital Shanghai Jiao Tong University School of Medicine. At the time of the STN-DBS surgery, the patient's head was fixed in a Leksell stereotactic headframe (Elekta, Stockholm, Sweden) under local anesthesia. Next, the electrodes used for DBS (Medtronic, Model 3387) were implanted under general anesthesia with computerized fluoroscopy. Lead placement in the STN motor area was planned and confirmed by a 1.5 Tesla magnetic resonance imaging (MRI) scan made after electrode implantation (Figure 1). After confirmation of the spatial location of the leads, an implantable pulse generator (IPG) (Medtronic, Minneapolis, MN; Model Activa PC, RC neurostimulator or Soletra [model 7426]) was connected to the DBS electrodes.

\section{DBS Programming}

DBS programming started 1 week after surgery. After a period of a few months of DBS parameter optimization, the clinically optimal treatment parameters were $60 \mu \mathrm{s}, 130 \mathrm{~Hz}$, and $1.5-2.0 \mathrm{~V}$ for pulse width, frequency, and amplitude, respectively. Our goal during this period was to obtain the greatest anticipated effect on dystonia, but below the threshold of inducing adverse side effects, such as dysarthria or bradykinesia. ${ }^{18}$ At 10 -year follow-up (10-yr FU), the pulse widths were $60-110 \mu$ s and the stimulator voltages were $2.15-3.50 \mathrm{~V}$ with frequencies in the range of $115-165 \mathrm{~Hz}$.

\section{Clinical Outcome Assessment}

Clinical assessments were performed at baseline, 1-year follow-up (1-yr FU), and 10-yr FU. The main clinical outcomes 


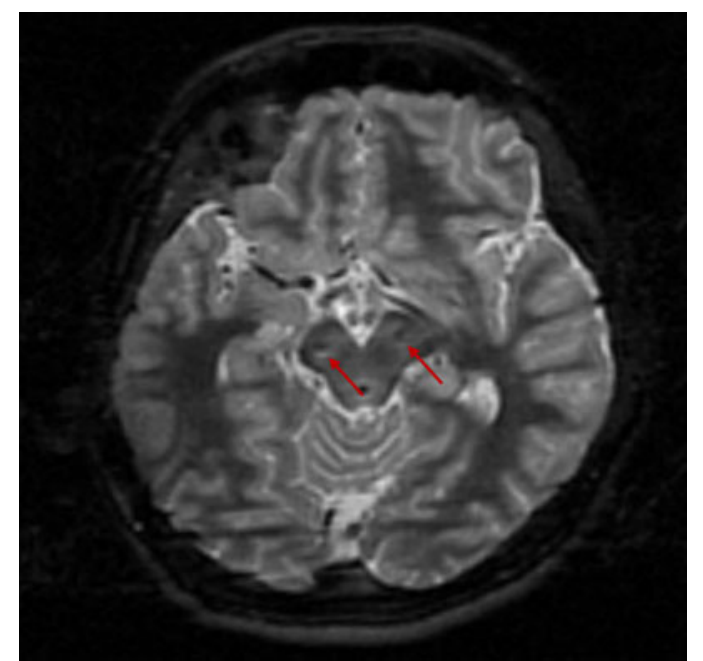

Figure 1: Representative postoperative brain magnetic resonance image (T2-weighted sequence) of one patient with implanted bilateral subthalamic nucleus DBS electrodes. The red arrows indicate the electrode locations at the subthalamic nucleus.

were the patient's dystonia severity and disability, as assessed by using retrospective video analyses of patient's dystonia with the Burke-Fahn-Marsden Dystonia Rating Scale (BFMDRS). The videos were rated by two independent clinical professionals. The mean of their clinical ratings was used for analysis. Three primary clinical outcome measures were derived from the BFMDRS: total scale score, motor scale score, and disability scale score. Also, motor sub-scores were obtained to examine dystonia in various body parts, including the eyes, mouth, speech/swallowing, neck, trunk, upper extremities, and lower extremities. Additionally, we collected qualitative information, if available, about the changes in patients' daily activities, psychosocial function, levels of anxiety and depression, and cognitive functioning from baseline to $10-\mathrm{yr}$ FU. Finally, adverse side effects, including surgery-related, devicerelated, and stimulation-related effects, were documented.

Additionally, data were collected on all patients regarding their age at disease onset, age at surgery (AS), disease duration (DD), and treatment history before surgery. Subsequently, we calculated the DD-AS ratio (DD/AS), normalizing DD according to AS, to estimate the proportion of lifetime lived with dystonia for each pediatric patient. ${ }^{19}$

\section{Statistical Analysis}

Data inspection showed that the BFMDRS scores (using the mean clinical ratings of the two observers) were not normally distributed. Therefore, we used a nonparametric test, namely the paired-sample Wilcoxon signed-rank test, to analyze the BFMDRS data. Specifically, three pairwise comparisons were made between the patients' data at baseline, 1-yr FU, and 10-yr FU, using a Bonferroni-adjusted level of statistical significance $(p=0.05 / 3=$ 0.0167 ) to control for an inflated false-positive error rate due to multiple testing. Additionally, a correlations analysis was conducted, using the Spearman's rank correlation coefficient, to assess at follow-up: (a) the relation of DD and AS with postoperative changes in BFMDRS scores and (b) the relation between the DD-AS ratio and postoperative changes in BFMDRS scores. Graphpad Prism 8 (GraphPad Software, Inc., San Diego, CA) was used for statistical analysis and plotting. In the text, quantitative variables are presented as mean \pm standard deviation and range, and qualitative variables as frequency and percentage.

\section{RESUlts \\ Patient Characteristics}

At the time of surgery, the patients (five women, four men) were aged between 7 and 21 years (mean age: $15.9 \pm 4.5$ years) and had been diagnosed with medically intractable isolated dystonia. All patients presented with generalized dystonia. They had tried pharmacotherapy initially but failed to show a clinical response. Two out of the nine patients were carriers of TORIA at the DYT-1 locus; the other seven patients tested negative. The age of dystonia onset ranged from 4 to 18 years (mean: $10.9 \pm 4.5$ years), the AS from 7 to 21 years (mean: $15.9 \pm 4.3$ years), and the DD from 1 to 12 years (mean: $5.0 \pm 3.6$ years). The mean ratio of $\mathrm{DD} / \mathrm{AS}$ was $0.32 \pm 0.19$. Clinical characteristics of each patient, including treatment history, are presented in Table 1 . The shorter term clinical outcomes of 5 patients (Patients 4, 5, 7, 8, and 9) have been partly reported previously. ${ }^{16}$

\section{Clinical Outcome: Dystonia Severity and Disability}

After STN-DBS, all patients experienced significant improvements in dystonia severity at 1-yr FU, which were maintained or became even larger at 10-yr FU (Table 1 and Figure 2). Specifically, the mean BFMDRS total score was improved by $75.3 \pm 15.4 \%(p=0.0039)$ at $1-\mathrm{yr} \mathrm{FU}$ and by $89.3 \pm 11.2 \%$ $(p=0.0039)$ at 10 -yr FU relative to the patients' scores obtained at baseline. Also, the mean improvement in the BFMDRS motor score was $77.1 \pm 16.6 \%$ (range: $44.9-92.9 \%$ ) at $1-\mathrm{yr}$ FU and $90.4 \pm 10.4 \%$ (range: $72.2-100 \%$ ) at 10-yr FU (Table 1). The mean improvements in the total score and motor score were significantly $(p=0.0039)$ larger at $10-\mathrm{yr} \mathrm{FU}$ than at 1 -yr FU (Figure 2C).

Similarly, the patients showed significant enduring improvements in their daily functioning (e.g., speech, swallowing, dressing, walking) after STN-DBS surgery. In particular, the mean BFMDRS disability score was improved by $69.5 \pm 13.6 \%$ (range: $45.5-89.5 \%$; $p=0.0039$ ) at 1 -yr FU and by $86.5 \pm 13.9 \%$ (range: $60.6-100 \%$; $p=0.0039)$ at $10-\mathrm{yr} \mathrm{FU}$ as compared with the mean disability score at baseline (Table 1). The mean improvement in the disability score at 10 -yrFU was significantly larger than the improvement gained at 1-yr FU (Figure 2C). Figure 2A and 2B depict for each patient his or her BFMDRS motor score and disability score observed at baseline and follow-up assessments. Table 2 summarizes for each patient the observed reductions from baseline to 10 -yr FU in dystonia and associated functional impairments.

After STN-DBS surgery, patients displayed significant reductions in the severity of dystonia in specific body parts, namely the neck $(n=6)$, upper extremities $(n=8)$, trunk $(n=9)$, and lower extremities $(n=9)$ at 1-yr FU and 10-yr FU. Except for the subscores related to the neck and upper extremities, the mean improvements in the sub-scores for dystonia in the trunk and lower extremities were significantly larger at 10-yr FU than 1-yr FU (Figure 2D). By comparison, dystonia in other body parts 


\section{Table 1: Patient characteristics and clinical outcomes}

\begin{tabular}{|c|c|c|c|c|c|c|c|c|c|c|c|c|c|c|c|}
\hline \multirow{3}{*}{ Patient/sex } & \multirow{3}{*}{$\begin{array}{c}\text { Age at } \\
\text { onset } \\
\text { (years) }\end{array}$} & \multirow{3}{*}{$\begin{array}{c}\text { Duration } \\
\text { of dystonia } \\
\text { (years) }\end{array}$} & \multirow{3}{*}{$\begin{array}{c}\text { Age at } \\
\text { surgery } \\
\text { (years) }\end{array}$} & \multirow{3}{*}{ DD/AS } & \multirow{3}{*}{ Genes } & \multirow{3}{*}{ Failed medication at surgery } & \multicolumn{9}{|c|}{ BFMDRS } \\
\hline & & & & & & & \multicolumn{3}{|c|}{ Baseline } & \multicolumn{3}{|c|}{ 1-yr FU } & \multicolumn{3}{|c|}{ 10-yr FU } \\
\hline & & & & & & & M & $\mathrm{D}$ & $\mathbf{T}$ & $\mathbf{M}$ & D & $\mathbf{T}$ & $\mathbf{M}$ & D & $\mathbf{T}$ \\
\hline $1 / \mathrm{M}$ & 4 & 3 & 7 & 0.43 & DYT-1 & $\begin{array}{l}\text { L-levodopa, benzhexol, carbamazepine, } \\
\text { clonazepam }\end{array}$ & 56 & 14 & 70 & 24 & 6 & 30 & 14 & 4 & 18 \\
\hline $2 / \mathrm{F}$ & 7 & 6 & 13 & 0.46 & DYT-1 & $\begin{array}{l}\text { Botulinum toxin, clonazepam, benzhexol, } \\
\text { haloperidol }\end{array}$ & 54.25 & 23 & 77.25 & 12.5 & 8 & 20.5 & 5 & 3.5 & 8.5 \\
\hline $3 / \mathrm{M}$ & 15 & 1 & 16 & 0.06 & - & L-levodopa, sodium valproate, benzhexol & 21 & 9.5 & 30.5 & 1.5 & 1 & 2.5 & 0 & 0 & 0 \\
\hline $4 / \mathrm{M}$ & 15 & 2 & 17 & 0.12 & - & Botulinum toxin, baclofen, trihexyphenidyl & 49.5 & 14 & 63.5 & 5.25 & 2.5 & 7.75 & 0 & 0 & 0 \\
\hline $5 / \mathrm{F}$ & 14 & 3 & 17 & 0.18 & - & Baclofen, tiapride, diazepam & 36.5 & 10 & 46.5 & 3.5 & 2.5 & 6 & 2.25 & 0.5 & 2.75 \\
\hline $6 / \mathrm{F}$ & 9 & 12 & 21 & 0.57 & - & tiapride, haloperidol & 51.25 & 16.5 & 67.75 & 28.25 & 9 & 37.25 & 14.25 & 6.5 & 20.75 \\
\hline $7 / \mathrm{F}$ & 10 & 10 & 20 & 0.50 & - & L-levodopa, botulinum toxin & 87 & 21 & 108 & 15.5 & 8 & 23.5 & 0 & 0 & 0 \\
\hline $8 / \mathrm{F}$ & 18 & 2 & 20 & 0.10 & - & Botulinum toxin, baclofen & 27 & 10 & 37 & 3 & 3 & 6 & 2 & 1.5 & 3.5 \\
\hline $9 / \mathrm{M}$ & 6 & 6 & 12 & 0.50 & - & Botulinum toxin, diazepam, baclofen & 45 & 19 & 64 & 13 & 4 & 17 & 5 & 3.5 & 8.5 \\
\hline Median & 10 & 3 & 17 & 0.43 & 1 & 1 & 49.5 & 14 & 64 & 12.5 & 4 & 17 & 2.25 & 1.5 & 3.5 \\
\hline IQR & $7-15$ & $2-6$ & $13-20$ & $0.11-0.50$ & I & I & $|36.5-54.25|$ & $10-19$ & $46.5-70.0$ & $3.5-15.5$ & $2.5-8.0$ & $6.0-23.5$ & $0-5.0$ & $0-3.5$ & $0-8.5$ \\
\hline
\end{tabular}

$\mathrm{F}=$ female; $\mathrm{M}=$ male; $\mathrm{DD}=$ duration of dystonia; $\mathrm{AS}=$ age at surgery; 1-yr FU = 1-year follow-up; 10-yr FU = 10-year follow-up; BFMDRS = Burke-Fahn-Marsden Dystonia Rating Scale;

$\mathrm{M}=$ motor score; $\mathrm{D}=$ disability score; $\mathrm{T}=$ total scores; $\mathrm{IQR}=$ interquartile range; $-=$ negative. 
Table 2: Overview of individual improvement of quality of life preoperatively and at the 10-yr follow-up

\begin{tabular}{|c|c|c|}
\hline Patient & Main disability preoperatively & Quality of life improvement at the 10-year follow-up \\
\hline 1 & $\begin{array}{l}\text { Unable to stand independently, tiptoe walk, falls occasionally, and the right hand } \\
\text { was clumsy with poor writing }\end{array}$ & Free walking with a few motor symptoms remaining \\
\hline 2 & $\begin{array}{l}\text { Difficulty holding a pen in the right hand; eating, and drinking only with help and } \\
\text { trouble falling asleep }\end{array}$ & $\begin{array}{l}\text { Free walking with limited incoordination and complete capacity for eating, drinking, } \\
\text { and writing independently }\end{array}$ \\
\hline 3 & Difficulty walking and school attendance suspended for half a year & Free walking but slowly with right full bow deformation and return to school \\
\hline 4 & Involuntary twisting of the neck severely affecting daily life & $\begin{array}{l}\text { Complete capacity for independent performance of activities of daily living and } \\
\text { could work normally }\end{array}$ \\
\hline 5 & $\begin{array}{l}\text { Severe tremors of the limbs cause difficulty in walking } \\
\text { and pain }\end{array}$ & Could work and socialize normally \\
\hline 6 & Dysgraphia, incapable of balanced walking, and clear speech & $\begin{array}{l}\text { Complete capacity for independent performance of activities of daily living with } \\
\text { minor difficulties and speech problems }\end{array}$ \\
\hline 7 & Difficulty in balance walking and clear speech & Walking, cycling freely, speaking fluently but a little weak \\
\hline 8 & Involuntary twisting of the neck resulting in difficulty eating and drinking & Return to daily life and no recurrence of symptoms even when the DBS was off \\
\hline 9 & $\begin{array}{l}\text { Serious impact on daily life and on learning, unable to walk and write, and school } \\
\text { attendance suspended }\end{array}$ & Able to walk and write normally but experiencing symptoms of depression \\
\hline
\end{tabular}
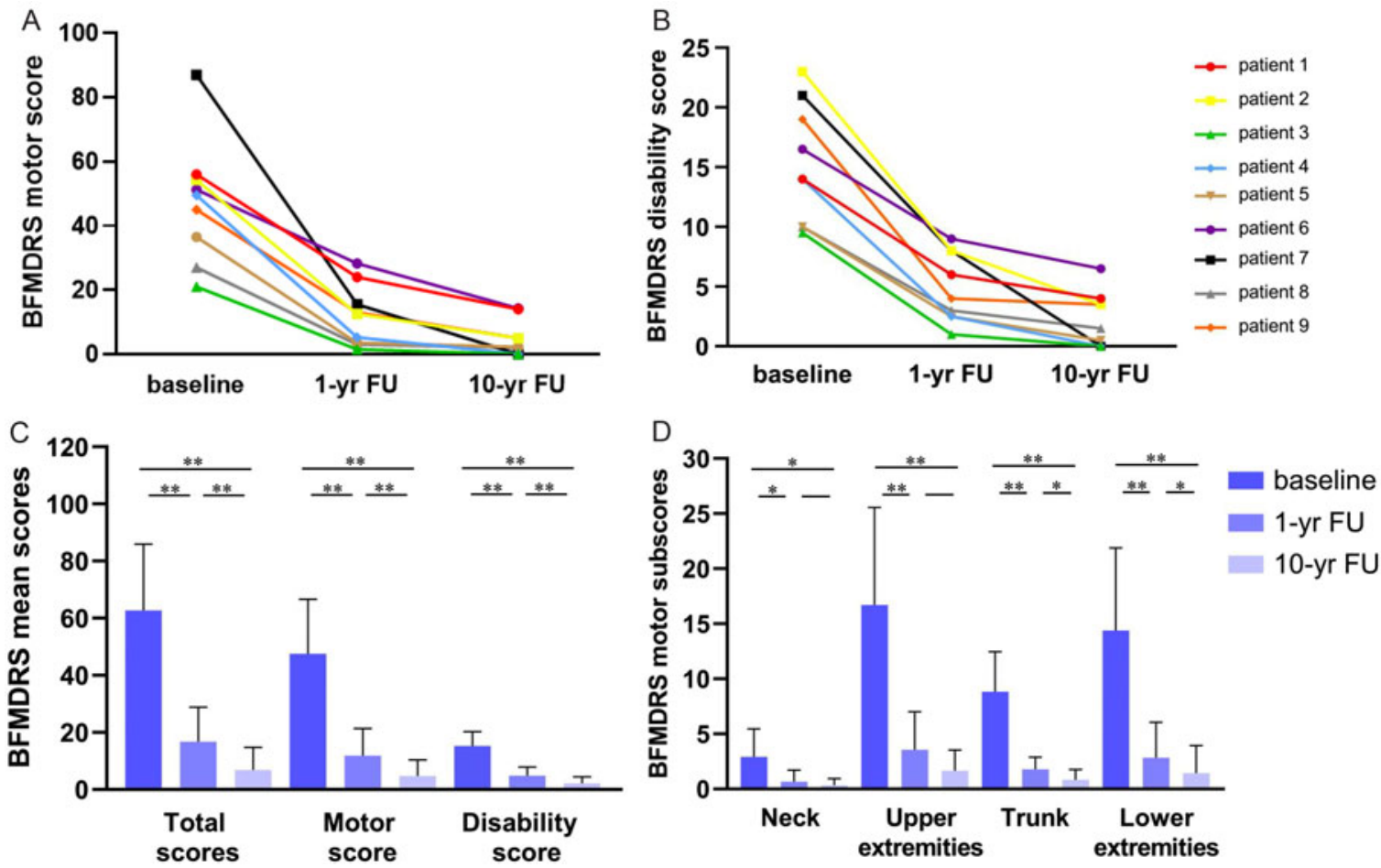

Figure 2: (A) Rating of individual BFMDRS motor scores for the 1-yr FU and 10-yr FU in all patients. (B) Rating of individual disability scores for the 1-yr FU and 10-yr FU in all patients. (C) Rating of BFMDRS mean motor score, disability score, and total score at baseline, 1-yr FU, and 10-yr FU. (D) Rating of BFMDRS motor sub-scores for the neck, upper extremities, trunk, and lower extremities at baseline, 1-yr FU, and 10-yr FU. Note: *, p <0.05; **, p <0.01.1-yr FU =1-year follow-up; 10-yr FU = 10-year follow-up; BFMDRS = Burke-Fahn-Marsden Dystonia Rating Scale.

improved less frequently (eyes, $n=1$; mouth, $n=2$; speech/ swallowing; $n=4$ ) during treatment. None of the patients developed dystonia in new parts of the body during the follow-up period.

\section{Correlation Analysis}

No significant correlation was detected between patients' DD and the improvements in BFMDRS scores achieved at 1-yr FU or 

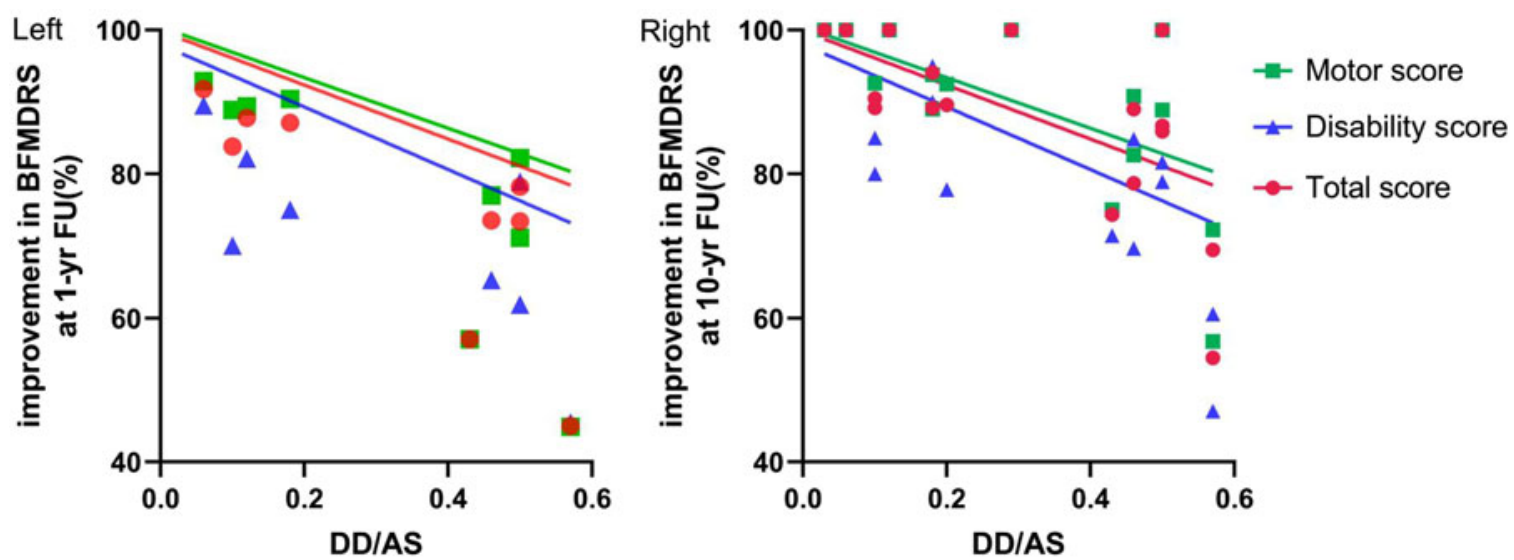

Figure 3: Left panel is a scatterplot of improvement in the Burke-Fahn-Marsden Dystonia Rating Scale (BFMDRS) scores at the 1-yr FU against the duration of disease $(D D)$ to age at surgery (DD/AS) ratio and negative correlations between the DD/AS ratio and motor $(\mathrm{p}=0.0124)$, disability $(\mathrm{p}=0.0549)$, and total scores $(\mathrm{p}=0.0096)$. Right panel is a scatterplot of improvement in BFMDRS scores at the 10-yr FU against the DD/AS ratio and negative correlations between the DD/AS ratio and motor $(\mathrm{p}=0.0150)$, disability $(\mathrm{p}=0.0351)$, and total scores $(\mathrm{p}=0.0150) . D D=$ duration of dystonia; AS =age at surgery; 1-yr FU =1-year follow-up; 10-yr FU =10-year follow-up; BFMDRS =Burke-Fahn-Marsden Dystonia Rating Scale.

10-yr FU. Neither was a significant correlation observed between AS and clinical outcome. However, significant correlations existed between the DD-AS ratio and the improvement in the BFMDRS total score and motor scale score observed at 1-yr FU (Figure 3A; Spearman's rank correlation coefficients for the motor, disability, and total scores were $-0.803,-0.670$, and -0.820 , respectively) and 10-yr FU (Figure 3B; Spearman's rank correlation coefficients for the motor, disability, and total scores were $-0.549,-0.485$, and -0.549 , respectively). The significant correlations reflect that as the time from the manifestation of the first dystonia symptoms to the initiation of STN-DBS treatment decreased, the improvement in patients' dystonia gained by the neurosurgical treatment was substantially larger.

\section{Adverse Events}

Generally, STN-DBS treatment was reasonably well tolerated by the patients. None of the patients experienced surgery-related adverse effects. The main device-related adverse effect was replacement of the IPG after battery expiration $(n=8)$. One patient experienced a breakage of the right electrode, which was replaced at the same anatomical location. Various stimulation-related adverse effects also occurred during treatment, although some could be resolved by DBS parameter adjustments. Stimulation-related adverse effects reported by patients at 10 -yr FU included swallowing difficulties/dysarthria $(n=1)$, gait disturbance $(n=4)$, dysarthria $(n=3)$, weakness $(n=2)$, vertigo $(n=1)$, anxiety $(n=1)$, depression $(n=2)$, and cognitive deficits $(n=2)$. One patient reported to have gained weight at 10 -year FU.

\section{Discussion}

In this study, we examined the short- and long-term clinical outcomes of nine patients who had undergone STN-DBS for medication-refractory pediatric isolated dystonia one decade ago in our hospital. After 1 year of STN-DBS treatment, all patients displayed marked reductions in overall dystonia severity (on average, about a 75\% reduction) and dystonia disability (70\% reduction). Moreover, the clinical improvements achieved at 1 -yr FU were persistent and turned out to be even significantly larger at 10 -yr FU (89\% reduction in overall dystonia severity and $87 \%$ in disability). The patients' body parts most favorably affected by STN-DBS were the neck, trunk, and the lower and upper extremities. Although most previous DBS treatment studies for pediatric dystonia have targeted the $\mathrm{GPi}^{9,}, 11,20$ some other studies have indicated that the STN could be a promising alternative DBS target for select pediatric patients with dystonia. ${ }^{21,22}$ The present study substantiates and extends the latter view and studies by indicating that STN-DBS could serve as a safe and effective alternative treatment option for at least some cases of medically intractable pediatric isolated dystonia. In this context, it is noteworthy that we also found that the clinical effectiveness of STN-DBS was even maintained in one patient when the battery became depleted and not replaced and, in another patient, when the stimulation was turned off. For the latter patient, extraction of the DBS brain implant is currently considered as the next therapeutic step. These latter observations suggest that STN-DBS treatment may induce functional brain changes that are enduring and preserved even without neuromodulation.

The patients' DD and AS did not display significant correlations with the improvements in dystonia severity or disability attained during the treatment. Interestingly, however, a significant inverse relationship was observed between the DD-AS ratio and the improvement in overall dystonia severity at 1-yr FU and 10-yr FU. That is, the shorter the pediatric patients lived with refractory dystonia before STN-DBS surgery, the larger were the clinical benefits they gained from the neurosurgical treatment at follow-up. If confirmed by further research, this finding raises the intriguing possibility that earlier STN-DBS intervention in patients with refractory pediatric isolated dystonia could greatly improve their short- and long-term clinical outcomes.

The STN-DBS treatment was reasonably well tolerated by the patients, but various adverse events were documented after surgery. Although some adverse side effects were transient, mild, or could be resolved by DBS parameters adjustment, other adverse effects were more serious and enduring. For example, two out of the nine patients reported to experience cognitive deficits, in particular impairment of executive functions, at $10-\mathrm{yr}$ FU. However, the main adverse event, occurring in eight patients, 
was device-related, namely replacing the IPG after battery expiration, often resulting in full or partial symptom remission and subsequent recovery after IPG replacement. These results are consistent with the findings from a multicenter study on adverse events in pediatric DBS treatment studies. ${ }^{20}$ In that study, 53 out of 72 pediatric patients ( $74 \%$ of the patient sample) experienced one or more adverse events during or after DBS surgery. Also, the most common adverse event, found in about $36 \%$ of the patients, was device-related and caused by battery depletion. Finally, after symptom recurrence and IPG replacement, the patients involved typically regained the clinical benefits from the DBS treatment. ${ }^{20}$ Thus, the risk-profile associated with pediatric STN-DBS in the present study seems to be comparable to that of other pediatric DBS treatment studies. Fortunately, the problems caused by battery depletion can nowadays be resolved by the use of new IPGs with rechargeable batteries, which reduces financial costs, reduces surgery-related health risks, and improves the patient's satisfaction with the DBS treatment, ${ }^{23}$ especially in pediatric patient populations. $^{24}$

Nonetheless, a major goal of pediatric and adult DBS treatment studies in general remains to reduce the risk of adverse side effects while not affecting or even improving the clinical benefits of DBS. One possible fruitful approach is to improve the accuracy of the surgical procedure and techniques used to target the DBS structure of interest. For example, a relationship has been identified in patients with Parkinson's disease between cognitive decline and both the position of the active contact within the STN and whether or not the electrode traverses the caudate nucleus, ${ }^{25}$ but whether this finding is relevant to STN-DBS treatment for pediatric dystonia needs to be verified. Similarly, a more precise targeting or the stimulation of a more restricted area in the STN has been found to reduce risk of adverse side effects in patients with Parkinson's disease, such as weight gain, depression, and cognitive deficits. At present, the exact targeting of a specific STN sub-region for DBS treatment remains a challenge, given its small size and the relative overlap in functional territories, even for the dorsolateral motor region of the STN. ${ }^{26-27}$

Several limitations of the present study should be acknowledged. First, the study was an observational study so that the presence of bias and confounding cannot be excluded. Second, we utilized a retrospective, and not a prospective, longitudinal design. Third, we focused on dystonia severity and disability as the primary clinical outcomes. At the time of the surgery, other clinical outcomes, such as cognitive function and quality of life, were not yet incorporated or systematically assessed in standard clinical outcome assessments at our hospital. Also, some researchers have raised concerns about the validity and reliability of the BFMDRS as a measure of dystonia symptoms and signs in children under the age of $16 .^{28}$ Fourth, the patient sample was small, which may have affected the statistical power to detect certain effects or correlations if truly present in the data. Finally, the patient sample was probably heterogeneous in terms of genetic etiology. ${ }^{29}$ We did not perform a whole-genome analysis but focused on the most common genes for isolated dystonia, including those at the DYT-1 and DYT-6 loci. Therefore, we cannot exclude the possibility that other genetic mutations might have played a role in the patients' dystonia and clinical response to STN-DBS treatment. As a consequence, it remains uncertain whether the present results can be generalized to the population of patients with pediatric isolated dystonia as a whole.

\section{Conclusion}

To the best of our knowledge, this is the first study to report on the clinical outcomes of a cohort of patients who underwent STN-DBS for pediatric isolated dystonia. The results provide supporting evidence for both the short- and long-term effectiveness and safety of STN-DBS treatment for pediatric isolated dystonia. Putative evidence is also provided that earlier STN-DBS interventions in patients with pediatric isolated dystonia are associated with better short- and long-term clinical outcomes. Given the study limitations, however, the present results should be considered preliminary until they are confirmed by further research. Comparative treatment studies are also required to characterize the relative effectiveness and safety profile of GPi DBS versus STN-DBS for refractory pediatric isolated dystonia.

\section{ACKNOWLEDGMents}

The authors thank all participants and the support from their families.

\section{Disclosure}

The authors report no conflicts of interest concerning the materials or methods used in this study or the findings specified in this paper.

\section{STATEMENT OF AUTHORShIP}

Conception and design: CZ, BS, DL, YW. Acquisition of data: HL, WX. Analysis and interpretation of data: WX, HL. Statistical review and critique: WX, HL, CZ, BS. Drafting the article: WX, HL. Final revision of the article: WX, HL, CZ, YW.

\section{REFERENCES}

1. Albanese A, Bhatia K, Bressman SB, et al. Phenomenology and classification of dystonia: a consensus update. Mov Disord. 2013;28:863-73. doi: 10.1002/mds.25475.

2. Lin JP, Lumsden DE, Gimeno H, et al. The impact and prognosis for dystonia in childhood including dystonic cerebral palsy: a clinical and demographic tertiary cohort study. J Neurol Neurosurg Psychiatry 2014;85:1239-44. doi: 10.1136/jnnp-2013-307041.

3. Lumsden DE, Gimeno H, Elze M, et al. Progression to musculoskeletal deformity in childhood dystonia. Eur J Paediatr Neurol. 2016;20:339-45. doi: 10.1016/j.ejpn.2016.02.006.

4. Luc QN, Querubin J. Clinical management of dystonia in childhood. Paediatr Drugs 2017;19:447-61. doi: 10.1007/ s40272-017-0243-3.

5. Vidailhet M, Vercueil L, Houeto JL, et al. Bilateral deep-brain stimulation of the globus pallidus in primary generalized dystonia. N Engl J Med. 2005;352:459-67. doi: 10.1056/NEJMoa 042187.

6. Park HR, Lee JM, Ehm G, et al. Long-term clinical outcome of internal globus pallidus deep brain stimulation for dystonia. PloS One 2016;11:e0146644. doi: 10.1371/journal.pone.0146644.

7. FitzGerald JJ, Rosendal F, de Pennington N, et al. Long-term outcome of deep brain stimulation in generalised dystonia: a series of 60 cases. J Neurol Neurosurg Psychiatr. 2014;85:1371-6. doi: 10.1136/jnnp-2013-306833.

8. Jinnah HA, Neychev V, Hess EJ. The anatomical basis for dystonia: the motor network model. Tremor Other Hyperkinet Mov (N Y) 2017;7:506. doi: 10.7916/d8v69x3s.

9. Krause P, Lauritsch K, Lipp A, et al. Long-term results of deep brain stimulation in a cohort of eight children with isolated dystonia. J Neurol. 2016;263:2319-26. doi: 10.1007/s00415-016-8253-6.

10. Miyagi Y, Koike Y. Tolerance of early pallidal stimulation in pediatric generalized dystonia. J Neurosurg Pediatr. 2013;12: 476-82. doi: 10.3171/2013.8.peds12578. 
11. Marks W, Bailey L, Reed M, et al. Pallidal stimulation in children: comparison between cerebral palsy and DYT1 dystonia. J Child Neurol. 2013;28:840-8. doi: 10.1177/0883073813488674.

12. Odekerken VJJ, Boel JA, Schmand BA, et al. GPi vs STN deep brain stimulation for Parkinson disease: three-year follow-up. Neurology 2016;86:755-61. doi: 10.1212/WNL.0000000000 002401.

13. Combs HL, Folley BS, Berry DTR, et al. Cognition and depression following deep brain stimulation of the subthalamic nucleus and globus pallidus pars internus in Parkinson's disease: a metaanalysis. Neuropsychol Rev. 2015;25:439-54. doi: 10.1007/ s11065-015-9302-0.

14. Wu YS, Ni LH, Fan RM, et al. Meta-regression analysis of the longterm effects of pallidal and subthalamic deep brain stimulation for the treatment of isolated dystonia. World Neurosurg. 2019;129: e409-16. doi: 10.1016/j.wneu.2019.05.165.

15. Cao C, Pan Y, Li D, et al. Subthalamus deep brain stimulation for primary dystonia patients: a long-term follow-up study. Mov Disord. 2013;28:1877-82. doi: 10.1002/mds.25586.

16. Deng Z, Pan Y, Zhang C, et al. Subthalamic deep brain stimulation in patients with primary dystonia: a ten-year follow-up study. Parkinsonism Relat Disord. 2018;55:103-10. doi: 10.1016/ j.parkreldis.2018.05.024.

17. Elkaim LM, Alotaibi NM, Sigal A, et al. Deep brain stimulation for pediatric dystonia: a meta-analysis with individual participant data. Dev Med Child Neurol 2019;61:49-56. doi: 10.1111/ dmcn.14063.

18. Koy A, Bockhorn N, Kuhn AA, et al. Adverse events associated with deep brain stimulation in patients with childhood-onset dystonia. Brain Stimul. 2019;12:1111-20. doi: 10.1016/ j.brs.2019.04.003.

19. Lumsden DE, Kaminska M, Gimeno H, et al. Proportion of life lived with dystonia inversely correlates with response to pallidal deep brain stimulation in both primary and secondary childhood dystonia. Dev Med Child Neurol. 2013;55:567-74. doi: 10.1111/ dmen.12117.

20. Rocha H, Linhares $\mathrm{P}$, Chamadoira $\mathrm{C}$, et al. Early deep brain stimulation in patients with myoclonus-dystonia syndrome.
J Clin Neurosci. 2016;27:17-21. doi: 10.1016/j.jocn.2015. 08.034 .

21. Benato A, Carecchio M, Burlina A, et al. Long-term effect of subthalamic and pallidal deep brain stimulation for status dystonicus in children with methylmalonic acidemia and GNAO1 mutation. J Neural Transm (Vienna) 2019;126:739-57. doi: 10.1007/ s00702-019-02010-2.

22. Barbosa B, Carra RB, Duarte KP, et al. Bilateral subthalamic nucleus stimulation in refractory status dystonicus. J Neurol Sci. 2018;388:159-61. doi: 10.1016/j.jns.2018.03.007.

23. Hitti FL, Vaughan KA, Ramayya AG, et al. Reduced long-term cost and increased patient satisfaction with rechargeable implantable pulse generators for deep brain stimulation. J Neurosurg. 2018:1-8. doi: 10.3171/2018.4.jns172995.

24. Pepper J, Zrinzo L, Mirza B, et al. The risk of hardware infection in deep brain stimulation surgery is greater at impulse generator replacement than at the primary procedure. Stereotact Funct Neurosurg. 2013;91:56-65. doi: 10.1159/000343202.

25. Witt K, Granert O, Daniels C, et al. Relation of lead trajectory and electrode position to neuropsychological outcomes of subthalamic neurostimulation in Parkinson's disease: results from a randomized trial. Brain 2013;136:2109-19. doi: 10.1093/brain/ awt151.

26. de Chazeron I, Pereira B, Chereau-Bodet I, et al. Impact of localisation of deep brain stimulation electrodes on motor and neurobehavioural outcomes in Parkinson's disease. J Neurol Neurosurg Psychiatry 2016;87:758-66. doi: 10.1136/jnnp-2015-310953.

27. Ostrem JL, Racine CA, Glass GA, et al. Subthalamic nucleus deep brain stimulation in primary cervical dystonia. Neurology 2011;76:870-8. doi: 10.1212/WNL.0b013e31820f2e4f.

28. Kuiper MJ, Vrijenhoek L, Brandsma R, et al. The BurkeFahn-Marsden Dystonia rating scale is age-dependent in healthy children. Mov Disord Clin Pract. 2016;3:580-6. doi: 10.1002/ mdc3.12339.

29. Aravamuthan BR, Waugh JL, Stone SS. Deep brain stimulation for monogenic dystonia. Curr Opin Pediatr. 2017;29:691-6. doi: 10.1097/mop.0000000000000548. 